\title{
EEG Evaluation of the Effects of Touching Intervention on Frustrating Tasks
}

\author{
Takashi MURAMATSU*, Yoshikazu WASHIZAWA*, \\ Carl B. BECKER ** and Kazuko HIYOSHI*** \\ * The University of Electro-Communications, 1-5-1, Chofugaoka, Chofu, Tokyo 182-8585, Japan \\ ** Kyoto University, Yoshida-honmachi, Sakyo-ku, Kyoto 606-8501, Japan \\ *** Yamato University, 2-5-1, Katayamacho, Suita, Osaka 564-0082, Japan
}

\begin{abstract}
Touching is a medical action wherein a practitioner touches a patient's body. Touching has positive physical and mental effects, and is an important technique for nursing. This study examines the effect of touching on patient frustration. To induce frustration, we adopted a mouse pointer-moving game and a calculation task: the game required moving a mouse pointer from the start to the goal without touching walls or obstacles. We asked the participants' acquaintance to gently touch their backs during the intervention trials. For evaluation, we used power spectral density (PSD) and electroencephalogram (EEG) event-related potential (ERP), and participants' self-evaluation scores. Theta, alpha, and beta band PSDs increased in frustrating tasks compared to the resting state, however, PSD increments of touching intervention tasks were less than that of control tasks. These results confirm that an acquaintance's touching can reduce frustration in difficult tasks, and concomitantly reduce unpleasant emotions.
\end{abstract}

Keywords: Touching, Frustrating emotion, Electroencephalography (EEG)

\section{INTRODUCTION}

The Japanese government is promoting home care of the elderly, not to mention staying at home to prevent the spread of COVID-19 [1]. Continually staying at home increases frustration even of healthy people, not only of care recipients. Care recipients are particularly liable to frustration when they face tasks that require more time or ability than they still have. Mental health requires simple home techniques to reduce frustration. Touching is one such medical action usable in home-care.

Since the 1970s, thousands of articles have reported on the effects of therapeutic touch in medicine, finding that gentle touch reduces stress $[2,3]$ and calms agitation and anger in nursing home residents and outpatients [4,5]. Objective scientific studies showed that touching reduced cardiac dysrhythmia [6] and patients' stress indicated by salivary amylase [7] or cortisol [8]. Chang also showed that touching has positive physical and mental effects in nursing settings [9]. Yucel et al. showed hand massage comforts and reduces anxiety of elderly people in nursing homes [10]. Han et al. showed back massage reduces pain and improves sleep quality of patients after gastrectomy [11]. Yamaguchi found touching reduced anxiety of child care in mothers [12].

But to our knowledge, no studies have used electroencephalograms (EEGs) to compare the effects of therapeutic touch on frustration or anger. We have focused on touching as a medical action to reduce frustration. In a previous study, we examined the effect of touching on unpleasant emotions induced by pictures, using not only self-evaluation, but also EEG late positive potential (LPP) and P300, known to be larger for emotional than for neutral stimuli [13,14]. We found that touching reduced LPP, P300, and valence responses to unpleasant pictures, concluding that touching reduces unpleasant emotions. However, patients' frustration is not caused by pictures, so this was insufficient to prove medical advantages of touch.

The purpose of this study is to examine the effect of touching on task-based frustration in settings close to nursing. In the nursing care field, patients and recipients feel frustrated when they are unable to perform the actions they want to do, or to finish their actions by their expected scheduled time. We used a mouse pointer moving game and a calculation task to reproduce frustration, evaluated effects using EEG power spectral density (PSD) and event related potential (ERP) as well as self-evaluation scores.

\section{METHODS}

\subsection{Task and Condition}

We used two tasks to induce frustrating emotions: (1) a mouse pointer-moving game, and (2) a calculation task. Using MIT Media Lab's free programming language Scratch, we created a game to generate frustration. The point of the game was to move a mouse pointer from start 
to goal without touching walls or moving obstacles for five minutes (Fig. 1 (a)). When the pointer touches walls or obstacles, the pointer explodes with visual and auditory effects, and the game restarts from the beginning. When the pointer reaches the goal, the stage is cleared, and the next stage starts. There were four stages to clear, but in our experiment, no one cleared all four stages in five minutes.

We created a time-pressure task using Tatsuno Information System's QuizGenerator. The task was to complete difficult mental calculations every three seconds for three minutes (Fig. 1 (b)). The subject chose an answer from among three options by mouse operation. To each response, loudspeaker sound effects further raised the tension.

The task (2) had six categories of ten questions each: four arithmetic operations, i) using two numbers of one or two digits; ii) using two numbers of one to three digits; iii) using two numbers of one to four digits; iv) using three numbers of one or two digits; v) using two numbers of one to five digits; and vi) using three numbers of one to three digits. Responses were stored using learningBOX provided by Tatsuno Information System Co., Ltd.

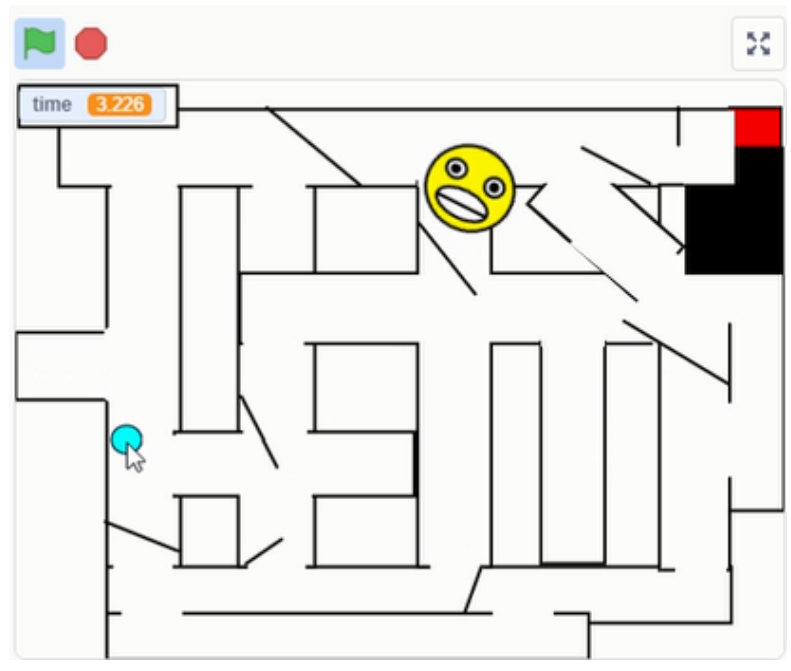

(a) Mouse pointer moving game

The blue ball is the pointer to be moved.

The start point is the left side of the figure, and the goal is the right side of the figure.

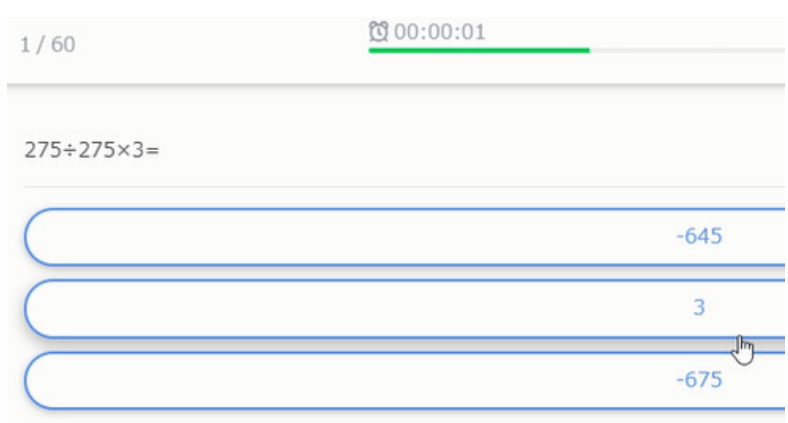

(b) Calculation program with time limit

Figure 1: Two tasks to induce frustration
For comparison, we used easier versions of the pointer game and the calculation task. The easy game (3) lacked obstacles and explosions, while the easy calculations (4) were simple one or two digit addition or subtraction, which everyone could master in the allotted time. This experiment was approved by Ethics committee for Experiments on Human Subjects, the University of Electro-Communications, and conducted in accordance with the approved research procedure, the relevant guidelines and regulations. Informed consent was obtained from all the subjects.

We recruited 18 healthy males $(25.0 \pm 3.87$ years old $)$ from the students of the University of Electro-Communications, for this experiment, envisioning that they would resemble frustrated care recipients, and a 24 year old male who would lay his hand on the back of the frustrated patient as a nurse might do. The man touching the subjects' backs was well acquainted with all the others, but had no real experience in nursing.

We used two trial conditions:

A) Normal: the frustrated subject was to react naturally with no intervention; and

B) Touch: another man gently lays his hands on the back of the frustrated subject.

The experimental procedure was as follows. First, we explained the conditions of the experiment, and secondly how to perform the required tasks. Then the subject carried out the task, after which he evaluated the task in terms of frustration (1: low-5: high), anger (1: low-5: high), and valence (1: pleasant-9: unpleasant). Each subject carried out six trials.

The following six tasks and conditions were randomly ordered for each subject: GC) control game with no intervention; GI) game with touching intervention; CC) control calculation with no intervention; CI) calculation task with touching intervention; GE) easy game with no intervention; CE) easy calculation with no intervention. Patterns GE and CE were carried out at the beginning or after the break to avoid interfering with the influence of unpleasant emotion in the other patterns.

Before the experiment, the subject rehearsed the task. There was a short break of at least one minute between trials, and a break of at least three minutes after three trials. The entire experiment took about 70 minutes: about 30 minutes for electrode installation and explanation, and about 40 minutes for the experiment itself.

\subsection{Environment}

Before the experiment, the position of a 27-inch LCD display screen was adjusted so that the subjects could easily 
view it. The screen alternately presented two windows: i) the screen instructing participants on the conditions, tasks, and self-evaluation (MATLAB); ii) the screen for carrying out the task (Google Chrome).

\subsection{EEG Measurement and Analysis}

For EEG recording, we used the MATLAB data acquisition toolbox, g.tec active EEG (g.GAMMAcap2, g.GAMMAbox, g.LADYbird), a TEAC BA1008 amplifier, and a Contec AI-1664 LAX-USB A/D converter. Before the trials, resting state 16-channel EEG was measured with subjects' eyes open and closed (three times of 10 seconds each, for a total of 60 seconds). The initial sampling rate was $1000 \mathrm{~Hz}$, and after recording, EEG was down-sampled to $500 \mathrm{~Hz}$. We analyzed PSD change rate and ERP in frontal (FP1, FP2, F5, F1, F2, F6), central (C1, C2), occipital (P5, P1, P2, P6, O1, O2), left temporal (T7), and right temporal (T8) areas. Trials with poorly measured EEG (voltage exceeding $100 \mu \mathrm{V}$ ) were excluded from the analysis. We applied a 1-30 Hz bandpass filter in ERP analysis, and calculated the grand mean waveforms when the mouse pointer exploded and when correct or incorrect answers were presented.

PSD change rate was calculated using the following equations for five bands: delta $(2-4 \mathrm{~Hz})$, theta $(4-8 \mathrm{~Hz})$, alpha $(8-12 \mathrm{~Hz})$, beta $(12-30 \mathrm{~Hz})$, gamma $(30-45 \mathrm{~Hz})$.

$$
\begin{aligned}
& \operatorname{PSD}\left[\mu V^{2} / H z\right]=\frac{\text { Power spectral in the band }\left[\mu V^{2}\right]}{\text { Bandwidth }[\mathrm{Hz}]} \\
& \text { PSD change rate } \\
& =\frac{P S D \text { in the latter half time of the task }\left[\mu V^{2} / \mathrm{Hz}\right]}{P S D \text { in resting state of open eyes }\left[\mu V^{2} / \mathrm{Hz}\right]}
\end{aligned}
$$

The latter half was defined as the time from the middle point to the end of the task.

\section{RESULTS}

\subsection{Self-Evaluation Scores}

Table 1 lists the result of self-evaluation scores. Unsurprisingly, frustration scores for the game and for the calculation task were significantly greater than those of

Table 1: Self-evaluation scores (mean \pm std)

\begin{tabular}{|l|l|c|c|c|}
\hline \multicolumn{1}{|c|}{ Task } & Condition & Frustration & Anger & Valence \\
\hline Game & Control & $3.61 \pm 1.14$ & $3.22 \pm 1.22$ & $5.89 \pm 1.91$ \\
\hline Game & Touching & $3.28 \pm 1.32$ & $3.06 \pm 1.39$ & $5.94 \pm 1.98$ \\
\hline Easy Game & Control & $1.83 \pm 0.857$ & $1.78 \pm 0.943$ & $3.61 \pm 2.06$ \\
\hline Calculation & Control & $3.11 \pm 1.28$ & $2.72 \pm 1.07$ & $5.89 \pm 2.08$ \\
\hline Calculation & Touching & $3.39 \pm 0.850$ & $2.83 \pm 1.20$ & $5.89 \pm 1.84$ \\
\hline Easy Calc. & Control & $1.39 \pm 0.979$ & $1.33 \pm 0.767$ & $2.50 \pm 1.69$ \\
\hline
\end{tabular}

the easy versions $\left(n=18\right.$, GC-GE: $p=1.51 \times 10^{-5}<0.05$, CC-CE: $\left.9.98 \times 10^{-6}<0.05\right)$; scores were unaffected by touching intervention.

\subsection{PSD Change Rate}

Figures 2-6 list the PSD change rates. Figure 7 shows the head topography of the Theta band PSD change rate.

\subsubsection{Delta Band PSD Change Rate}

Figure 2 shows that in the frontal, central, occipital, and left temporal areas, the delta band PSDs in the control game (GC) increased compared with the resting state, whereas the delta PSDs in the game with intervention (GI) and the easy game (GE) were unchanged. For the calculation task, the delta band PSDs during the tasks increased in all areas compared with the resting state for all cases (CC, CI, and CE), whereas there is no significant difference among the conditions.

\subsubsection{Theta Band PSD Change Rate}

Figure 3 shows that in the frontal, central, occipital, and left temporal areas, the theta band PSDs in the control game (GC) increased compared with the resting state, whereas the theta PSDs in the game with intervention (GI) and the easy game (GE) were unchanged.

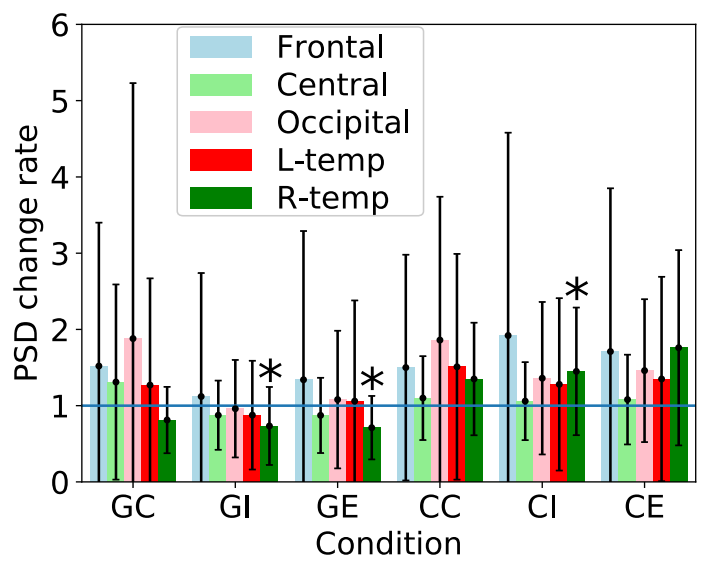

Figure 2: Delta band PSD change rate (*: significant difference between mean and $1(\mathrm{p}<0.05, \mathrm{n}=18))$

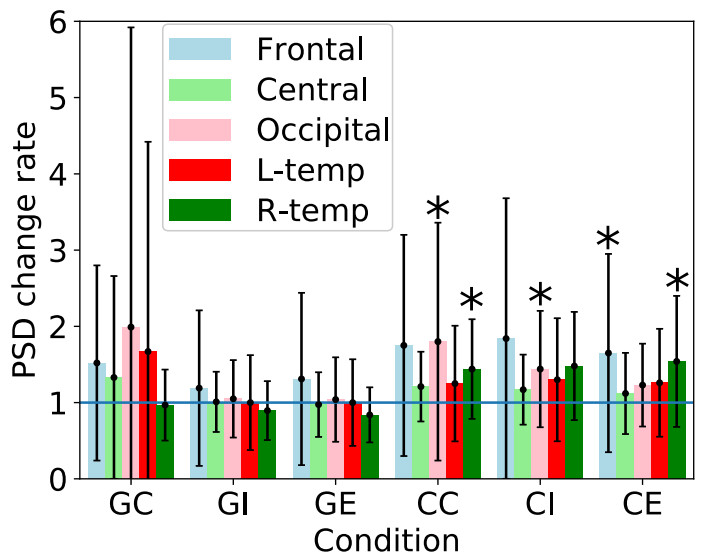

Figure 3: Theta band PSD change rate (*: significant difference between mean and $1(\mathrm{p}<0.05, \mathrm{n}=18))$ 


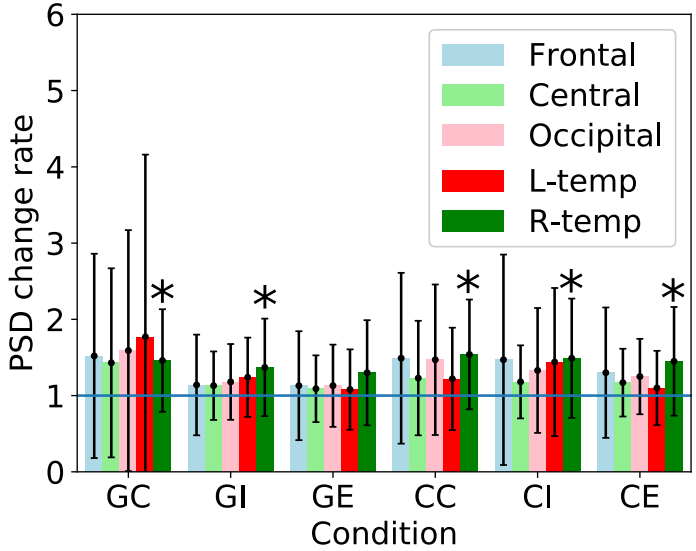

Figure 4: Alpha band PSD change rate

$(*$ : significant difference between mean and $1(\mathrm{p}<0.05, \mathrm{n}=18))$

Figure 7 shows that the theta band PSD in the control game (GC) increased across the entire cerebellum, especially, the right occipital area. However, the theta band PSD in the intervention game (GI) was unchanged; its topography resembled that of the easy game (GE).

From Fig. 3, both control calculation tasks (CC and CE) increased compared to the resting state. The occipital theta band PSD change rate in the calculation control task (CC) was greater than those in calculations with intervention (CI) and the easy calculation task (CE).

Figure 7 shows that the theta band PSD of the calculation control group (CC) increased across the entire head, but especially in frontal and occipital areas. However, in the occipital area, the theta band PSD change rate in the calculation intervention group (CI) was smaller than that in the control group (CC), and the topography in the intervention group (CI) resembled that of the easy calculation task (CE).

\subsubsection{Alpha Band PSD Change Rate}

Figure 4 shows that in all areas, the alpha band PSDs in the control game (GC) increased compared with the resting state, whereas the alpha PSDs in the game with intervention (CI) and the easy game (GE) were unchanged. For the calculation task, the alpha band PSDs during the tasks increased in all areas compared with the resting state for all cases (CC, CI, and CE), whereas there is no significant difference among the conditions.

\subsubsection{Beta Band PSD Change Rate}

Figure 5 shows that in all areas, the beta band PSDs in the control game (GC) increased compared with the resting state, and its beta band PSD change rates were greater than those of the game with intervention (GI) and the easy game (GE). It is known that anger increases the beta band PSD in the frontal area [15]. In our experiment, not only in the frontal area but also in the right temporal area, the beta band PSD was increased

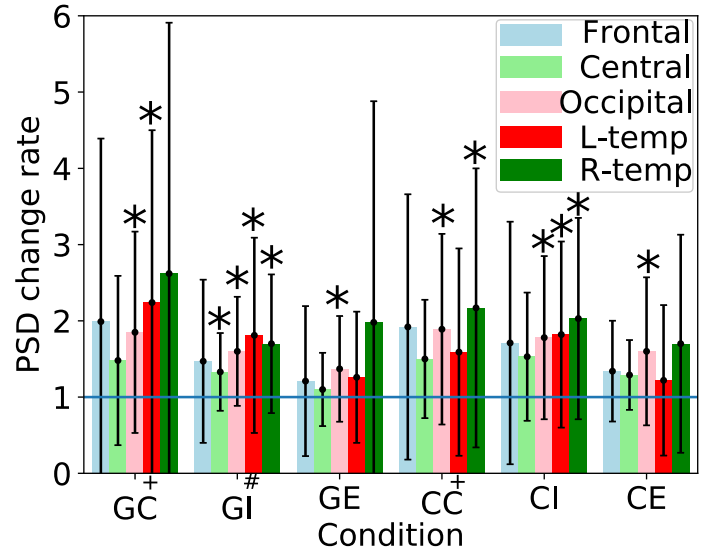

Figure 5: Beta band PSD change rate (*: significant difference between mean and 1 $(\mathrm{p}<0.05, \mathrm{n}=18,+\mathrm{n}=17, \#: \mathrm{n}=16))$

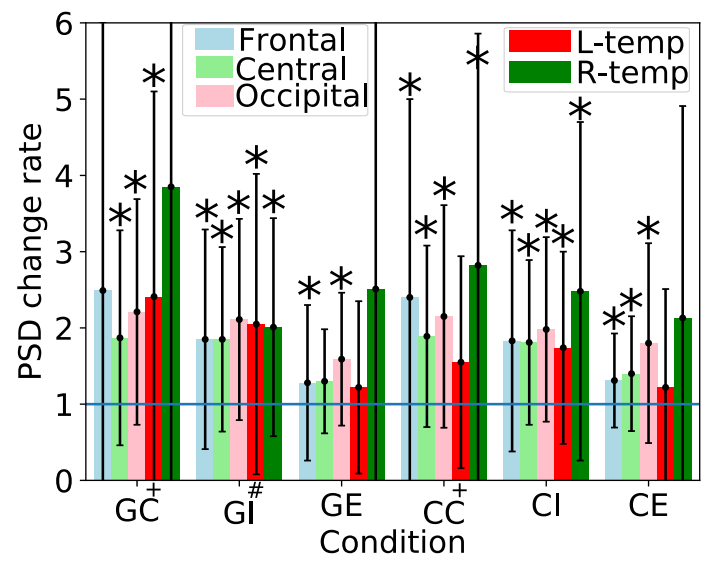

Figure 6: Gamma band PSD change rate (*: significant difference between mean and 1 $(\mathrm{p}<0.05, \mathrm{n}=18,+: \mathrm{n}=17, \#: \mathrm{n}=16))$

by frustration evoked by the tasks ( $\mathrm{GC}$ and $\mathrm{CC}$ ). The frontal beta band PSD change rate in the control calculation task (CC) was greater than those in the calculation with intervention group (CI) and the easy calculation group (CE).

\subsubsection{Gamma Band PSD Change Rate}

Figure 6 shows that across all areas, the gamma band PSDs in the control game (GC) increased compared with the resting state, and this score was higher than that of those in the intervention game (GI) and easy game (GE). In the frontal and temporal areas, the gamma band PSDs in all calculation tasks increased over the resting state; in particular, the frontal and right temporal areas showed greater gamma band PSD change rates in the control calculation task (CC) than in the calculation with intervention group (CI) and the easy calculation group (CE).

\subsection{ERP}

Figure 8 shows the grand mean waveforms in the frontal area (P300s were also observed on the other electrodes). 

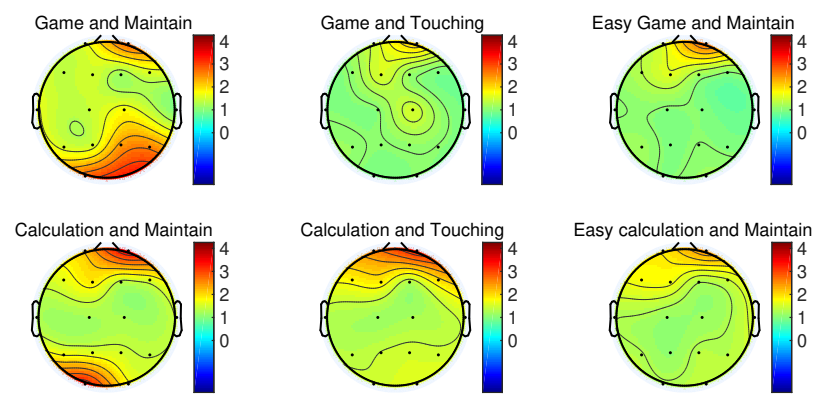

Figure 7: Head topography of theta band PSD change rate

The minimum and average numbers of subjects were 7 and 14.8 (resp.).
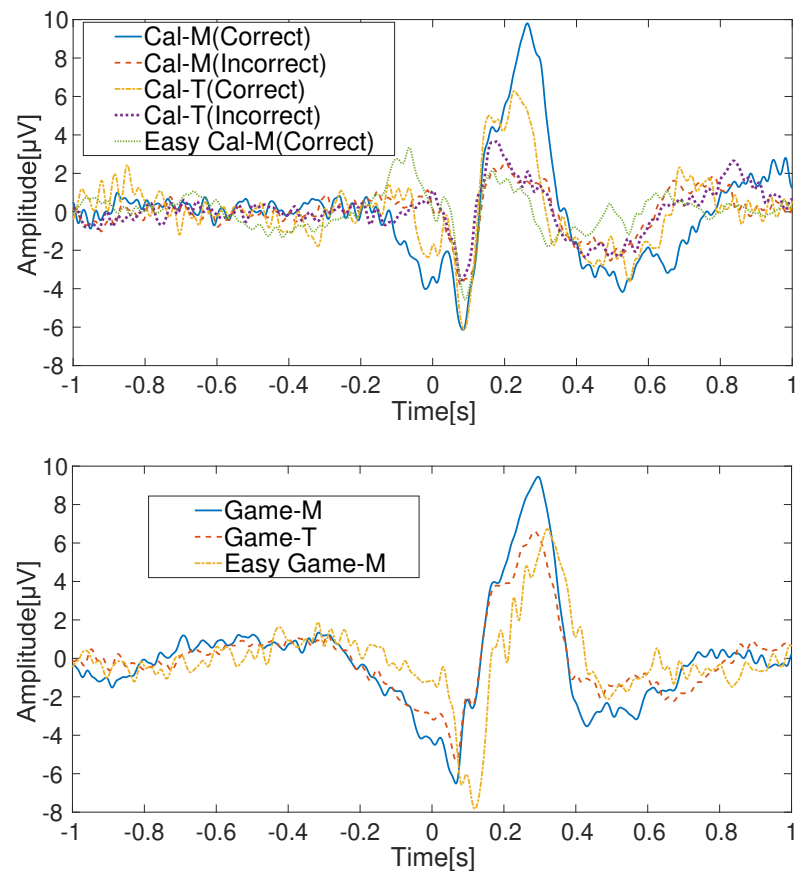

Figure 8: Grand mean waveforms in frontal area (Cal: calculation, M: Control, T: touching):

The number of subjects: 18, except for Easy Game-M: 17. The minimum numbers of trials and channels per subject are Game: 16, and Calculation 10, and average number are Game: 158, Calculation: 140.

The grand mean waveform of incorrect answers in the easy calculation task was not calculated because the sample number was small. We set the onset of ERP to the explosion in the pointer game, and to the mouse click response in the calculation task. P300 was observed 100-400 milliseconds after each onset.

To compare individual differences in P300, we calculated each subject's peak amplitude using the following procedure. First we smoothed the waveforms with a $4 \mathrm{~Hz}$ low-pass and determined the P300 peak from the smoothed waveforms. Then we treated the mean potential in the 100 milliseconds around the peak as P300 peak amplitude. Table 2 shows the means and standard deviations of the P300 peak amplitudes.
Table 2: P300 peak amplitudes $[\mu \mathrm{V}]($ mean \pm std)

\begin{tabular}{|l|c||c|c|c|c|c|}
\hline \multicolumn{2}{|l|}{ Condition } & Frontal & Central & Occipital & Left-Temp. & Right-Temp. \\
\hline \hline GC & & $6.76 \pm 5.05$ & $8.91 \pm 4.82$ & $5.49 \pm 3.17$ & $4.92 \pm 3.46$ & $4.39 \pm 4.14$ \\
\hline GI & & $6.13 \pm 5.60$ & $7.08 \pm 3.40$ & $3.83 \pm 1.62$ & $3.64 \pm 1.95$ & $3.35 \pm 1.57$ \\
\hline GE & & $4.80 \pm 4.22$ & $7.29 \pm 4.46$ & $3.79 \pm 3.41$ & $3.77 \pm 2.41$ & $3.71 \pm 2.41$ \\
\hline CC & C & $6.59 \pm 4.66$ & $4.40 \pm 2.45$ & $2.44 \pm 1.09$ & $3.93 \pm 2.37$ & $2.90 \pm 1.36$ \\
\hline CI & C & $4.98 \pm 6.38$ & $4.09 \pm 2.79$ & $2.39 \pm 1.17$ & $2.61 \pm 2.49$ & $2.41 \pm 2.08$ \\
\hline CE & C & $1.77 \pm 2.78$ & $1.15 \pm 1.34$ & $0.971 \pm 0.649$ & $0.898 \pm 1.43$ & $1.31 \pm 1.22$ \\
\hline CC & I & $2.66 \pm 2.54$ & $2.51 \pm 2.06$ & $1.56 \pm 0.975$ & $1.33 \pm 1.15$ & $1.90 \pm 1.43$ \\
\hline CI & I & $2.55 \pm 2.73$ & $2.23 \pm 2.07$ & $1.53 \pm 1.27$ & $1.50 \pm 1.39$ & $1.94 \pm 1.65$ \\
\hline
\end{tabular}

From Table 2, in all areas, the P300 peak amplitudes in the control game (GC) were greater than those in the game with touching (GI) and the easy game (GE). Except for the frontal area, the P300 peak amplitudes in two patterns showed no difference in GI and GE. In both control (CC) and intervention $(\mathrm{CI})$, correct answers in calculation showed significantly greater peak $\mathrm{P} 300$ amplitudes for the difficult tasks than for easy calculation tasks (CE) $(n=17$, CC-CI: $\mathrm{p}=8.34 \times 10^{-5}<0.05$, CI-CE: $\mathrm{p}=0.0317<0.05$ ). When subjects calculated incorrectly, touching intervention did not affect their P300 peak amplitudes.

\section{DISCUSSION}

As predicted, difficult games and calculations engendered significantly greater frustration than easy versions (GEandCE) $\left(\mathrm{n}=18\right.$, GC-GE: $1.51 \times 10^{-5}<0.05$, CC-CE: $9.98 \times 10^{-6}<0.05$ ). Thus our model can examine frustration of patients unable to perform what is expected of them in limited time-frames.

Unsurprisingly, correct responses to difficult calculations (CC) showed significantly greater P300 peak amplitudes than those to easy calculations (CE), suggesting that subjects felt more sense of accomplishment in correctly making difficult calculations.

PSD change rates and peak P300 amplitudes showed the effects of touching.

All games raised PSD over the resting state, consistent with a previous study [15] in which video clips of anger and joy increased frontal, central, and occipital theta, frontal beta, and frontal gamma, band PSDs. But in this touching intervention study, the PSD of touching intervention games (GI) and easy games (GE) was less than that of control games (GC), suggesting that touching lowers frustration levels during difficult tasks, especially in (i) occipital and left-temporal area of theta, alpha, and beta bands; and (ii) left- and right-temporal area of beta and gamma bands.

P300 amplitudes for the control game (GC) were greater than for the intervention games (GI) and easy games (GE), 
which showed no significant difference except for the frontal area. These results also show that touching reduced unpleasant emotion.

Although some results of EEG scores suggested that touching reduced unpleasant emotion, self-evaluation scores did not show significant difference between control and touching conditions. The reduction of unpleasant emotion by touching occurs subconsciously.

Curiously, touching intervention affected neither self-evaluation scores nor P300 peak amplitudes in any calculation tasks (CC, CI, CE). This needs further examination concerning what frustration tasks are affected by touching.

\section{CONCLUSION}

This study examined the effect of a touching intervention on people feeling frustration from time pressure or inability to perform a task, which many nursing home patients may feel. A "nurse" laying his hands on the back of the "patients" feeling frustration significantly lowered the patients' anger and frustration.

Future research needs not only to increase our sample size, but also to examine the effects of touching by female nurses, on female patients, and at first meeting, rather than between acquaintances. Furthermore, experiments in the real nursing field are needed.

\section{ACKNOWLEDGEMENTS}

This work was supported by a Research Grant from the KDDI Foundation.

\section{REFERENCES}

1. Ministry of Health, Labour and Welfare; https:// www.mhlw.go.jp/stf/seisakunitsuite/bunya/hukushi_ kaigo/kaigo_koureisha/chiiki-houkatsu/ (accessed 2021.11.01). (in Japanese)

2. Kramer, D.; Energetic modalities as a self-care technique to reduce stress in nursing students, Journal of Holistic Nursing, 36(4), pp.366-373, 2018.

3. Bukowski, E. L.; The use of self-Reiki for stress reduction and relaxation, Journal of Integrative Medicine, 13(5), pp.336-340, 2015.

4. Waze, C., Leathard, H. L., Grange, J., Tiplady, P., and Stevens, G.; Evaluation of healing by gentle touch, Public Health, 119(1), pp.3-10, 2005.

5. Gillis, K., Lahaye, H., Dom, S., Lips, D., Arnouts, H., and Bogaert, P. V.; A person-centred team approach targeting agitated and aggressive behaviour amongst nursing home residents with dementia using the Senses Framework, International Journal of Older People Nursing, 14(4), e12269, 2019.

6. Zolfaghari, M., Eybpoosh, S., and Hazrati, M.; Effects of therapeutic touch on anxiety, vital signs, and cardiac dysrhythmia in a sample of iranian women undergoing cardiac catheterization: A quasiexperimental study, Journal of Holistic Nursing, 30(4), pp.225-234, 2012.

7. Wilkinson, D. S., Knox, P. L., Chatman, J. E., Johnson, T. L., Barbour, N., Myles, Y., and Reel, A.; The clinical effectiveness of healing touch, Journal of Alternative and Complementary Medicine, 8(1), pp.33-47, 2002.

8. Woods, D. L., Beck, C., and Sinha, K.; The effect of therapeutic touch on behavioral symptoms and cortisol in persons with dementia, Forschende Komplementärmedizin / Research in Complementary Medicine, 16(3), pp.181-189, 2009.

9. Chang, S. O.; The conceptual structure of physical touch in caring, Journal of Advanced Nursing, 33(6), pp.820-827, 2001.

10. Yücel, Ş. C., Arslan, G. G., and Bagci, H.; Effects of hand massage and therapeutic touch on comfort and anxiety living in a nursing home in Turkey: A randomized controlled trial, Journal of Religion \& Health, 59(1) pp.351-364, 2020.

11. Han, M.-S., and Lee, K.-Y.; The effect of back massage on degree of pain, state anxiety, and quality of sleep of postoperative patients with gastrectomy, Asian Oncology Nursing, 12(1), pp.69-76, 2012.

12. Yamaguchi, H.; Aibu: Hito no kokoro ni fureru chikara, NHK Publishing, 2003. (in Japanese)

13. Muramatsu, T., Washizawa, Y., Hiyoshi, K.; Touching effects on negative visual perception: Quantitative evaluation of emotion regulation using EEG, The 6th Annual Meeting of The Society for Nursing Science and Engineering, 2018. (in Japanese)

14. Mini, A., Palomba, D., Angrilli, A., and Bravi, S.; Emotional information processing and visual evoked brain potentials, Perceptual and Motor Skills, 83(1), pp.143-152, 1996.

15. Aftanas, L. I., Reva, N. V., Savotina, L. N., and Makhnev, V. P.; Neurophysiological correlates of induced discrete emotions in humans: An individually oriented analysis, Neuroscience and Behavioral Physiology, 36(2), pp.119-130, 2006. 


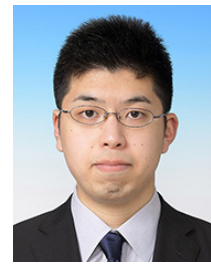

Takashi MURAMATSU (Non-member)

Takashi Muramatsu was a graduate student of the University of Electro-Communications, Japan. He was mainly interested in EEG and psychology, and had been studying the effects of touch care by EEG for more than two years. In March 2020, he completed his master's degree in Department of Computer and Network Engineering from this university. After April 2020, he is currently working for Atom Medical Corporation, in the research and development of medical devices in obstetrics and gynecology. In particular, he is working on the development of fetal monitoring systems and devices.

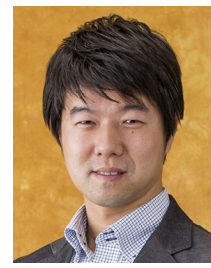

\section{Yoshikazu WASHIZAWA (Non-member)}

Yoshikazu Washizawa is an Associate Professor of The University of Electro-Communications, Japan. He received the B.E. degree from the Nagoya Institute of Technology, Nagoya, Japan, in 2002, and the M.E. and Ph.D. degrees from the Tokyo Institute of Technology, Tokyo, Japan, in 2004 and 2008, respectively. He was with TOSHIBA Corporation, Tokyo, from 2004 to 2005. In 2005, he was with the Tokyo Institute of Technology as a JSPS Research Fellow. From 2005 to 2011, he was with the Laboratory for Advanced Brain Signal Processing, Brain Science Institute, RIKEN, Saitama, Japan. Since 2011, he has been with the University of ElectroCommunications, Tokyo. His current research interests include pattern recognition, machine learning, and biomedical signal processing. He is a member of IEEE and IEICE.

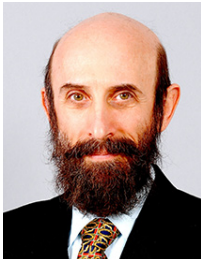

Carl B. BECKER (Non-member)

Carl B. Becker is Professor of Medical Ethics in the Policy Science Unit of Kyoto University, Japan. His latest work examines psychosocial support for medical professionals, patients, and bereaved families. His Japanese and English books and articles on death and dying, ethics and environment are available online.

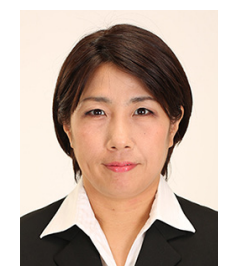

\section{Kazuko HIYOSHI (Non-member)}

Kazuko Hiyoshi is a Professor in the Faculty of Health Science of Yamato University, Japan. She researches care of patients using EEG and fMRI. Recently, her interests have moved to improving health care for Japanese women. 